\title{
Implantes unitários com carga imediata: possibilidade de reabilitação oral e estética
}

\section{- uma revisão de literatura}

\author{
Immediate load unit implants: possibility of oral and aesthetic rehabilitation - literature review \\ Implantes unidades de carga inmediata: posibilidad de rehabilitación oral y estética -revisión de la
}

\section{Resumo}

A perda dentária acontece por diversas razões e traz consequências ruins para a saúde bucal do indivíduo, incluindo transtornos sociais e estéticos, configurando-se em um desafio para a área odontológica. $\mathrm{O}$ uso de implantes para melhorar a função, estética e saúde bucal avançou a partir do conceito de osseointegração, evoluiu de protocolos convencionais com duas fases cirúrgicas para implantação imediata após a exodontia, inclusive com carga imediata, possibilitando a reabilitação oral de edêntulos totais, parciais e de um único elemento. O objetivo deste estudo foi realizar uma revisão de literatura sobre as possibilidades de reabilitação nas reabilitações unitárias com implantes em situação de carga imediata. A pesquisa foi realizada através de um levantamento bibliográfico de artigos, dissertações, periódicos e publicações sobre implantes unitários com carga imediata, indexados em bases de dados Scielo, Pubmed, Medline, Google Scholar, num período entre os anos de 2015 a 2020. A literatura é ampla e demonstra altas taxas de sucesso e previsibilidade de implantes com carga imediata, sendo um procedimento muito benéfico para o paciente devido à redução de tempo total de tratamento, redução de custos e resultados bastante satisfatórios, inclusive quando se trata de estética e satisfação do paciente. É possível realizar implantes imediatos e reabilitação imediata mesmo em áreas infectadas, com segurança e efetividade, desde que siga todos os cuidados necessários para cada situação, tanto no procedimento cirúrgico como ainda no cuidado do paciente pós cirurgia. Em todos os casos, o uso de implantes com carga imediata requer planejamento cuidadoso.

Palavras-chave: Implantes dentários; Carga imediata em implante dentário; Perda dentária unitária; Prótese dentária; Implantodontia.

\begin{abstract}
Tooth loss happens for several reasons and brings bad consequences for the individual's oral health, including social and aesthetic disorders, constituting a challenge for the dental field. The use of implants to improve function, esthetics and oral health advanced from the concept of osseointegration, evolved from conventional protocols with two surgical phases for immediate implantation after extraction, including immediate loading, enabling the oral rehabilitation of total and partial edentulous and a single element. The aim of this study was to conduct a literature review on the possibility of rehabilitation in unit rehabilitation with implants under immediate load. The research was conducted through a bibliographic survey of articles, dissertations, journals and publications on unit implants with immediate loading, indexed in Scielo, Pubmed, Medline, Google Scholar databases, in a period between the years 2015 to 2020. Literature it is broad and demonstrates high success rates and predictability of implants with immediate loading, being a very beneficial procedure for the patient due to the reduction of total treatment time, cost reduction and very satisfactory results, including when it comes to aesthetics and patient satisfaction. patient. It is possible to perform immediate implants and immediate rehabilitation even in infected areas, safely and effectively, as long as you follow all the necessary precautions for each situation, both in the surgical procedure and in the care of the patient after surgery. In all cases, the use of implants with immediate loading requires careful planning.
\end{abstract}

Keywords: Dental implants, immediate loading; Single tooth loss; Dental prosthesis; Implantology. 


\begin{abstract}
Resumen
La pérdida de dientes ocurre por varios motivos y trae consigo malas consecuencias para la salud bucal del individuo, incluidos los trastornos sociales y estéticos, lo que constituye un desafío para el campo odontológico. El uso de implantes para mejorar la función, la estética y la salud bucal avanzó desde el concepto de osteointegración, evolucionado a partir de protocolos convencionales con dos fases quirúrgicas para la implantación inmediata después de la extracción, incluida la carga inmediata, permitiendo la rehabilitación oral de desdentados totales y parciales y de un solo elemento. El objetivo de este estudio fue realizar una revisión de la literatura sobre la posibilidad de rehabilitación en rehabilitación unitaria con implantes en situaciones de carga inmediata. La investigación se realizó a través de un relevamiento bibliográfico de artículos, disertaciones, revistas y publicaciones sobre implantes unitarios con carga inmediata, indexados en las bases de datos Scielo, Pubmed, Medline, Google Scholar, en un período comprendido entre los años 2015 a 2020. La literatura es amplia y demuestra altos índices de éxito y previsibilidad de implantes con carga inmediata, siendo un procedimiento muy beneficioso para el paciente debido a la reducción del tiempo total de tratamiento, reducción de costos y resultados muy satisfactorios, incluso en lo que se refiere a la estética y satisfacción del paciente. Es posible realizar implantes inmediatos y rehabilitación inmediata incluso en zonas infectadas, de forma segura y eficaz, siempre que se sigan todas las precauciones necesarias para cada situación, tanto en el procedimiento quirúrgico como en el cuidado del paciente tras la cirugía. En todos los casos, el uso de implantes con carga inmediata requiere una planificación cuidadosa.
\end{abstract}

Palabras clave: Implantes dentales, carga inmediata; Pérdida de un solo diente; Prótesis dental; Implantología.

\title{
1. Introdução
}

A perda dentária acomete o homem por diversas razões. Doenças periodontais e cáries, são causas predominantes destas perdas, o que altera a função mastigatória, e o osso residual perdendo sua função, irá se remodelar alterado densidade e massa, reduzindo a altura e volume ósseo, prejudicando a saúde bucal e estética (Silva \& Almeida, 2017).

Para autores como Assis e Araújo (2016) e Batista (2019) a perda dentária configurou-se ao longo do tempo, como desafio na área odontológica. O uso de implantes dentários a partir de estruturas de titânio para servirem de apoio à prótese dentária foram avanços nesta área, que iniciou com a descoberta da osseointegração e avançou ao longo do tempo, para tentar resolver os problemas das próteses convencionais que não satisfaziam todas as necessidades do paciente, inclusive acarretando alguns problemas (Assis \& Araújo, 2016; Batista, 2019).

O protocolo inicial de Brånemark a partir do conceito de osseointegração, consistia em duas etapas distintas. Posteriormente, novos estudos permitiram a reabilitação em apenas uma etapa, iniciando-se o conceito de carga imediata em implantes dentários. A carga imediata em implantes dentários foi aplicada primeiramente em pacientes com perdas dentárias totais, mais tarde, para os parcialmente desdentados, e mais recentemente passou a ser utilizada em implantes unitários, e em áreas estéticas (Batista, 2019).

Alguns autores afirmam que os implantes podem ser instalados após a extração dentária, juntamente com a provisionalização de carga imediata. Esta é uma técnica bem aceita atualmente na implantodontia, devido as suas vantagens nas reabilitações orais, principalmente pela redução do tempo de tratamento e reposição dos dentes perdidos, conseguindo de forma mais rápida resultados funcionais e estéticos ao paciente, com altas taxas de sucesso (Tanaka Junior, 2015; Farro, 2017; Silva \& Almeida, 2017; Bitar Junior, 2020).

Conforme Felice et al. (2015) implantes imediatos são aqueles implantes instalados imediatamente instalados após a extração do dente, ou seja, em um alvéolo fresco. A principal vantagem do procedimento é encurtar a duração do tratamento, não precisando esperar a cicatrização parcial dos tecidos mole, que leva entre 2 a 6 semanas, ou para consolidação óssea em torno de 4 a 6 meses (Felice et al., 2015)

Como explicam Costa (2018), Grandi et al. (2105), Felice et al. (2015) e Guideti et al. (2015) existem indicações e contraindicações para o uso de implantes osseiontegrados. Estas referem-se diretamente ao paciente e suas condições de saúde geral, hábitos e expectativas. Contraindicações também dizem respeito ao profissional, seu treinamento ou falta dele e sua experiência com implantes (Costa, 2018; Grandi et al., 2015; Felice et al., 2015; Guideti et al., 2015).

Assis e Araújo (2016) acrescentam que para um resultado satisfatório com implantes, é importante obedecer às 
corretas indicações: travamento primário, boa condição óssea, escolha adequada do implante, condição sistêmica satisfatória do paciente, ausência de maus hábitos como tabagismo e bruxismo.

Para diversos autores, diferenças significativas em termos de perda óssea marginal, complicações, aspectos de partes moles, resultado estético e satisfação do paciente são alguns parâmetros comparados entre carregamento convencional e carregamento imediato de implantes de dentes unitários, assim como altos índices de previsibilidade tanto nas regiões anterior e posterior (Den Hartog et al., 2016, Tonetti et al., 2017; Heydecke et al., 2018; Ayna et al., 2018; Grandi et al., 2015; Felice et al., 2015; Lucas et al., 2019).

Mesmo em sítios infectados, a sobrevida dos implantes imediatos pode ser bastante satisfatória e apresentar taxas de sobrevivência próximas dos implantes colocados em locais sadios, desde que incluídos vários procedimentos ao tratamento como: a assepsia e antissepsia, prescrição adequada de antibióticos sistêmicos profiláticos, desbridamento minucioso do alvéolo após extração dental minimamente invasiva, e gerenciamento eficaz da carga imediata até a restauração definitiva (Bitar Júnior et al., 2020; Falcon-Guerrero \& Falcon-Pasapera, 2020; Sampaio, 2020).

Desta forma, realizou-se este estudo no sentido de obter informações para a utilização da técnica de carga imediata sobre implantes unitários a fim de contribuir para ampliar e disseminar o conhecimento sobre o tema, importante para o aperfeiçoamento profissional. Tratou-se de uma revisão de literatura realizada através de um levantamento bibliográfico de artigos, dissertações, periódicos e publicações sobre implantes com carga imediata, indexados em bases de dados Scielo, Pubmed, Medline, Google Scholar, com período de publicação entre os anos de 2015 a 2020.

\section{Metodologia}

Tratou-se de uma revisão de literatura narrativa, desenvolvida nos meses de janeiro a junho de 2021.

A busca por publicações científicas foi realizada entre fevereiro e março de 2021, utilizando-se de publicações científicas obtidas nas seguintes bases de dados: Scielo, Pubmed, Medline e Google Scholar. Foram utilizados os termos "implantes dentários" e "carga imediata" isoladas ou combinadas através dos operadores booleanos "and" e "or", com período de publicação entre os anos de 2015 a 2020.

Foram estabelecidos como critérios de inclusão: artigos científicos, dissertações e trabalhos de conclusão de curso publicados nos idiomas (português, inglês e espanhol), nos anos de 2015 a 2020, disponíveis em formato completo. Em relação aos critérios de exclusão estabeleceu-se pela eliminação de estudos duplicados, que não apresentassem relação com o tema ou relevância científica e publicações anteriores ao ano de 2015.

Inicialmente analisou-se os resumos e posteriormente leitura do texto completo para seleção dos estudos. Ao final foram incluídas 30 publicações para a leitura criteriosa e categorização destes, sendo os resultados discutidos segundo a visão de diversos autores.

\section{Revisão e Discussão da Literatura}

Conforme Batista (2019) o protocolo inicial de Brånemark a partir do conceito de osseointegração, consistia em duas etapas distintas. Posteriormente, a reabilitação pode ocorrer em apenas uma etapa, iniciando-se o conceito de carga imediata em implantes dentários a partir dos anos de 1980. A carga imediata em implantes dentários foi aplicada primeiramente em pacientes com perdas dentárias totais, mais tarde, expandiu-se para os parcialmente desdentados, vindo a ser mais recentemente utilizada em implantes unitários em áreas estéticas (Batista, 2019).

Vários estudos apontam que os implantes realizados imediatamente após a extração dentária juntamente com a provisionalização de carga imediata é uma técnica bem aceita atualmente na implantodontia, devido as suas vantagens nas reabilitações orais, principalmente pela redução do tempo de tratamento e reposição dos dentes perdidos, conseguindo de 
forma mais rápida, resultados funcionais e estéticos ao paciente, com altas taxas de sucesso (Tanaka Júnior, 2015; Farro, 2017; Silva \& Almeida, 2017; Bitar Júnior, 2020).

Matiello e Trentin (2015) relataram em caso clínico fatores que influenciam na osseointegração de implantes com carga imediata. Na mesma sessão foram realizadas a exodontia e a instalação de implante dentário com carga imediata com o objetivo de manter a saúde, a estética dos tecidos periodontais, a função mastigatória e o conforto da paciente. Os resultados clínicos foram favoráveis e condizentes com a técnica utilizada. A altura óssea e gengival foi preservada, resultando em estética favorável da prótese fixa definitiva. O planejamento interdisciplinar foi essencial para a obtenção do resultado.

Grandi et al. (2015) realizaram um estudo com cento e cinco pacientes com necessidade de uma única coroa suportada por implante. Os pacientes foram distribuidos em gupos, sendo 35 pacientes tratados com carga imediata, 35 com carga precoce e 35 pacientes receberam carga convencional. Implantes imediatos ou precoces foram inseridos com torque superior a $45 \mathrm{Ncm}$ e receberam coroas temporárias não oclusivos, enquanto os implantes carregados convencionalmente foram diretamente restaurados com coroas definitivas. As coroas provisórias foram substituídas por definitivas após 4 meses. As medidas de resultados avaliadas foram a sobrevivência dos implantes, complicações e alterações do nível ósseo marginal. Não houveram diferenças significativas para qualquer uma das medidas de resultado entre as três estratégias de carregamento após 1 ano. Nenhuma grande diferença clínica foi observada em relação à sobrevivência do implante ou complicações e alterações do nível ósseo marginal ao carregar implantes únicos imediatamente, cedo ou convencionalmente.

Felice et al. (2015) compararam a eficácia de implantes unitários instalados imediatamente pós extração com implantes colocados em alvéolos preservados após 4 meses de cicatrização, e acompanhamento de 1 ano. As medidas de resultado avaliadas foram falhas de implantes, complicações, estética, usando o escore estético rosa (PES), nível de osso marginal peri-implantar mudanças e satisfação do paciente, registradas por avaliadores cegos. Nove (36\%) implantes não foram carregados imediatamente no grupo imediato contra 19 (76\%) implantes no grupo de colocação tardia, porque um torque de inserção superior a $35 \mathrm{Ncm}$ não foi obtido. Dois pacientes desistiram 4 meses após o carregamento no grupo tardio. Dois implantes falharam no grupo imediato (8\%) contra nenhum no grupo tardio. Três complicações menores ocorreram no grupo imediato e duas no grupo tardio. Os níveis ósseos marginais no implante após enxerto ósseo foram 0,01 mm para implantes imediatos e 0,06 mm para implantes tardios, revelando uma diferença estatisticamente significativa. A satisfação do paciente foi avaliada após 4 meses e um ano após o carregamento, todos ficaram totalmente satisfeitos tanto para função quanto para estética, afirmando que se submeteriam ao mesmo procedimento novamente. Não foram observadas diferenças significativas entre os dois procedimentos, embora dois implantes falharam no grupo pós-extrativo imediato. Parece mais difícil obter um torque de inserção do implante superior a $35 \mathrm{Ncm}$ em alvéolos preservados com osso derivado de algas após um período de cura de 4 meses do que em locais pós-extrativos imediatos.

Guideti et al. (2015) avaliaram a sobrevivência de implantes dentários individuais submetidos à função imediata de doze pacientes com áreas edêntulas na região posterior de mandíbula. Foi instalado pelo menos um implante de 3,75 mm x 11 $\mathrm{mm}$ ou 3,75 mm x $13 \mathrm{~mm}$ (plataforma regular). Parâmetros clínicos e radiográficos foram utilizados como métodos de avaliação. Após 12 meses, a taxa de sobrevida foi de 83,35\%. Os implantes não apresentaram mobilidade clínica, com valores de ISQ (OSSTELL) próximos a 70, perda óssea de até $2 \mathrm{~mm}$ e profundidade de sondagem menor ou igual a $3 \mathrm{~mm}$. Apesar da região posterior da mandíbula ser uma área na qual os implantes de carga imediata devem ser colocados com cautela, este tratamento apresentou boa taxa de sucesso na amostra estudada. A carga funcional imediata de implantes unitários na área posterior da mandíbula foi considerada uma técnica confiável. É importante que o paciente não apresente bruxismo, que possua osso suficiente para o apoio e que uma boa higiene oral seja praticada de modo a alcançar uma boa taxa de sobrevida.

Tanaka Júnior (2015) relatou um caso clínico que foi usado implante unitário com carga imediata em região estética apresentando as suas vantagens, indicações, limitações, desvantagens e complicações da carga imediata em implante unitário. 
Após a fixação adequada do implante, a localização deste foi moldada e no mesmo dia foi parafusado um provisório. A oclusão foi aliviada até que o contato fosse mínimo em todos os movimentos excursivos, propiciando que a reabilitação estética/funcional e a osseointegração do implante fossem um processo simultâneo e favorável um ao outro. Foi alcançada a devolução imediata da estética e parte da função dentária, sem interferir no processo de osseointegração, permitindo que a paciente pudesse manter suas atividades sociais habituais. Implantes unitários com carga imediata são seguros se respeitados os pré-requisitos operatórios e reabilitadores.

Matsumoto et al. (2016) relataram um caso de exodontia de incisivo lateral com instalação imediata de implante, enxertia de tecido mole, colocação de biomaterial e provisionalização, com o objetivo de manutenção estética. Os resultados clínicos imediatos, após uma semana e com dois anos de uso da prótese definitiva mostraram-se satisfatórios do ponto de vista estético e funcional. Destaca-se a importância da realização de planejamento adequado para seleção e momento da execução de procedimentos clínicos necessários para o sucesso do tratamento.

Andreiuolo et al. (2016) apresentaram um caso clínico de paciente de 65 anos com necessidade de implante imediato pós-exodontia na região anterior da maxila em que o protocolo de tratamento clínico consistiu em diagnóstico da necessidade de exodontia, sondagem óssea, cirurgia reversa em modelo de gesso para a confecção de um pilar de zircônia e uma restauração provisória. Após seis meses foi realizada a moldagem do pilar preparado para a confecção da restauração definitiva. Os resultados obtidos preencheram os requisitos estéticos, funcionais e biológicos em função dos corretos diagnósticos, planejamento e execução do caso.

Den Hartog et al. (2016) compararam o carregamento imediato ao carregamento convencional após 5 anos de implantes de dentes unitários anteriores colocados em locais cicatrizados num total de 62 pacientes com perda de um dente anterior superior. Os pacientes foram tratados aleatoriamente com um implante restaurado com uma coroa temporária não oclusiva no prazo de 24 horas após a colocação do implante (grupo imediato) ou de acordo com um procedimento de dois estágios após 3 meses (grupo convencional). Todos os implantes foram colocados em locais cicatrizados. Visitas de acompanhamento foram realizadas após a entrega da coroa definitiva após 1 e 5 anos do procedimento. As medidas de resultado analisados foram alterações radiográficas do nível ósseo marginal, sobrevivência do implante, complicações, aspectos dos tecidos moles (profundidade de sondagem, placa, sangramento, alterações do nível do tecido mole), resultado estético e satisfação do paciente. Três pacientes em cada grupo de estudo perderam o acompanhamento. Não foram encontradas diferenças significativas em termos de perda óssea marginal em nenhum dos grupos, complicações, aspectos de partes moles, resultado estético e satisfação do paciente. Quanto à sobrevivência apenas um implante foi perdido no grupo imediato. $\mathrm{O}$ resultado da carga imediata não é inferior à carga convencional para implantes de dentes unitários anteriores colocados em locais cicatrizados.

Assis e Araújo (2016) discorreram sobre indicações, vantagens e desvantagens da técnica de carga imediata aplicada em implantodontia, analisando os critérios atuais desta técnica. O uso da carga imediata é uma realidade em implantodontia, e pode ser realizada de forma segura com altas taxas de sucesso. Dentre as vantagens evidenciadas por este método pode-se destacar, a satisfação do paciente com rápida execução e conclusão da terapia, restituindo a estética, melhorando a autoestima e satisfação. Para um resultado satisfatório, é importante obedecer às corretas indicações quais sejam o travamento primário, boa condição óssea, escolha adequada do implante, condição sistêmica satisfatória do paciente, ausência de maus hábitos como tabagismo, bruxismo e apertamento, são requisitos para o sucesso da técnica aplicada.

Miguel Júnior et al. (2016) apresentaram caso de paciente do sexo feminino, 32 anos de idade com insatisfação estética frente aos elementos 22 e 23, confirmada no exame de tomografia computadorizada volumétrica cone beam, uma reabsorção óssea extensa, provavelmente sendo associada aos anos de ortodontia relatados pela paciente. Foram indicadas a exodontia associada a enxerto de tecido conjuntivo e colocação imediata de coroas provisórias. No caso apresentado foi 
alcançado um resultado favorável do ponto de vista estético e funcional. A técnica aplicada mostrou-se segura e eficaz, salientando-se que é necessário um planejamento adequado, envolvendo avaliação tomográfica, domínio de técnica cirúrgica e protética, para se obter um bom resultado. Os implantes instalados imediatamente após a exodontia devem, obrigatoriamente, possuir estabilidade inicial no momento da implantação, como requisito para o sucesso clínico. O preenchimento do espaço alveolar deve ser realizado com o propósito de ser evitada uma possível reabsorção óssea da parede alveolar, comprometendo o resultado final. A utilização de próteses livres de metal é uma opção viável, do ponto de vista estético e funcional, suprindo a necessidade do paciente, devendo o cirurgião-dentista estar atento aos componentes protéticos disponíveis pelo sistema de implante escolhido.

Silva e Almeida (2017) descreveram sobre reabilitação com implantes dentários imediatos, seguidos de reabilitação imediata e os principais aspectos desta abordagem, além de vantagens e desvantagens e suas aplicações práticas. O implante imediato apresenta vantagens como tempo de tratamento reduzido, reabsorção óssea minimizada melhorando a função e a estética a partir da primeira cirurgia. Os implantes imediatos nem sempre poderão ser executados, é necessária avaliação criteriosa para realização do procedimento e execução dentro de um protocolo específico para que sua taxa de sucesso seja elevada.

Tonetti et al. (2017) realizaram um estudo randomizado com 124 pacientes sendo distribuidos em dois grupos onde 62 pacientes receberam implantes imediatos e 62 receberam implantes atrasados (12 semanas após a extração). Um implante foi perdido por infecção no grupo de implante imediato e oito pacientes adicionais não conseguiram cumprir com o acompanhamento. Ambos os procedimentos foram bem tolerados pelos pacientes e associados a altos níveis de satisfação do paciente. Implantes imediatos exigiram aumento ósseo no momento da colocação com mais frequência do que implantes retardados (72\% vs 43,9\%). O fechamento primário ideal foi obtido com mais frequência em implantes retardados, que também eram menos propensos a exibir falha da ferida. As profundidades de sondagem em torno dos implantes imediatos foram maiores do que aquelas observadas em implantes retardados no momento da inserção da coroa e aos 12 meses. Os implantes imediatos mostraram uma tendência para níveis mais elevados de perda óssea radiográfica durante o acompanhamento de 36 meses. No entanto, essas diferenças podem ser confundidas por uma colocação mais profunda de implantes imediatos na hora da cirurgia. Os implantes imediatos resultaram em uma recessão marginalmente menor do tecido mole em torno dos dentes adjacentes $0,3 \mathrm{~mm}$ vs $0,5 \mathrm{~mm}$, mas esse achado não atingiu significância estatística. As pontuações PES de 12 meses foram mais frequentemente consideradas inadequadas em casos de implantes imediatos do que tardios (42\% vs $19 \%)$.

Farro (2017) realizou uma revisão sistemática de artigos científicos relativos a implantes imediatos com carga imediata na reabilitação oral. Segundo o estudo, a reabilitação de pacientes desdentados é um processo complexo, em que devem ser considerados aspectos fisiológicos, patológicos e psicológicos. Fatores como o envelhecimento, a alteração do fluxo salivar, a redução da coordenação motora, a reabsorção óssea do rebordo, a fragilidade do tecido mucoso, as alterações dos tecidos de suporte e a adaptação psicológica interferem e têm de ser considerados quando se planeja uma reabilitação oral implanto- suportada. Quando conjugados com diferentes opções clínicas de reabilitação oral implanto-suportada, constituem soluções estéticas e funcionais viáveis, proporcionando inúmeros benefícios para a reabilitação de pacientes edêntulos. A demanda estética de implantes imediatos em carga imediata é cada vez mais uma solução à qual o profissional tem de estar preparado para saber gerir.

Costa (2018) identificou os tópicos iniciais a serem observados e seguidos para realizar uma reabilitação oral usando implantes. Os implantes osseointegrados são indicados em casos de edentulismos parciais e totais, confecção de elementos unitários, edentulismos com distribuição desfavorável ou número insuficiente de pilares naturais para próteses fixas, insatisfação ou rejeição às próteses totais ou parciais removíveis. As contraindicações podem ser divididas em absolutas e 
relativas. As contraindicações absolutas, estão relacionadas com condições que podem afetar potencialmente a saúde geral do paciente e comprometer seriamente a sobrevivência dos implantes. Em geral, as doenças sistêmicas não controladas contraindicam qualquer tipo de cirurgia. As contraindicações relativas são situações em que há um risco adicional para a ocorrência de complicações e fracassos se o tratamento for efetuado nesse momento, quando se eliminam essas situações, cessam as contraindicações. São consideradas contraindicações relativas, as doenças sistêmicas sobre controle, medicação crônica, radioterapia localizada sobre a região edêntula, pacientes em crescimento, gravidez, expectativas não-realísticas sobre o tratamento, falta de treinamento ou experiência do profissional, bruxismo, higiene oral inadequada, hábitos como fumar e beber. Pacientes que fazem uso de nicotina, álcool, ou drogas, podem ter um efeito negativo sobre vascularização do sítio cirúrgico exigindo cautela ao planejar implantes para esses indivíduos. Após avaliação do paciente e definição da reabilitação com implantes, o planejamento deve seguir algumas etapas sendo a primeira delas a anamnese do paciente, exame radiográfico, protocolo fotográfico, exame extra e intra bucal, utilização de modelos de estudos montados em articulador em relação cêntrica, movimentação ortodôntica, enceramento diagnóstico e guia cirúrgico. Um planejamento reverso bem executado é o maior indicativo para o sucesso do tratamento reabilitador com implantes.

Em um ensaio clínico prospectivo multicêntrico Heydecke et al. (2018) trataram 94 pacientes com 99 implantes, destes, oitenta e oito implantes compareceram ao acompanhamento de 3 anos. Ocorreu uma perda óssea inicial entre inserção do implante em 6 meses (- 0,92 $\pm 1,23 \mathrm{~mm})$, níveis ósseos estabilizados de 6 meses a 3 anos $(0,13 \pm 0,94 \mathrm{~mm})$ sem mudança significativa. A taxa de sobrevivência cumulativa de 3 anos foi de $98,9 \%$ e a taxa de sucesso cumulativa foi de $96,9 \%$. Pontuações do índice de papila de 2 ou 3 foram observadas em 88,6\% dos locais na visita de 3 anos em comparação com $32,8 \%$ na inserção do implante. Melhorias foram observadas para todos os outros resultados, incluindo sangramento na sondagem, estética, placa e perfil de impacto na saúde bucal. O protocolo restaurador foi associado a alta estabilidade primária, satisfação do paciente, níveis ósseos estáveis e um melhora geral dos resultados dos tecidos moles em um período de 3 anos sendo o tratamento apresentado como uma opção viável para restaurações unilaterais de dentes anteriores superiores e prémolares com resultados clínicos bem-sucedidos de curto a médio e longo prazo.

Araújo et al. (2018) realizaram um estudo seccional com o objetivo de caracterizar áreas edêntulas de pacientes candidatos à reabilitação com um implante dentário osseointegrado unitário em região estética da maxila por meio de TCFC correlacionando os parâmetros a seguir: distância da JCE até a CO, distância entre a CO e distância entre as CD dos dentes adjacentes ao EP, largura e altura óssea do EP, espessura do palato na região dos segundos pré-molares e molares bem como a espessura horizontal e vertical do tecido mole na região do EP. Os resultados foram analisados por meio de estatística descritiva e do teste de Correlação de Spearman. Correlações fracas e moderadas e sem diferenças estatísticas significativas foram encontradas na maioria das comparações $(\leq 0,29 \mathrm{e} \geq 0,30 \leq 0,49$, respectivamente). Apenas para a correlação espessura da mucosa do EP versus distância entre JCE e CO no dente mesial ao EP foi encontrada diferença estatística significativa ( $\mathrm{p}=$ $0,03)$ e correlação moderada $(r=0,56)$. TCFC é uma ferramenta viável para a região maxilofacial, pois permitiu a mensuração de diversos parâmetros de tecido mole e duro da cavidade bucal úteis para o planejamento e previsibilidade de tratamento reabilitador com implantes dentários.

Ayna et al. (2018) fizeram um estudo com 63 pacientes que receberam implantes curtos $(6 \mathrm{~mm})$ para substituição de um único dente na maxila posterior. Quarenta pacientes foram submetidos ao conceito funcional imediato, enquanto 15 dos implantes foram carregados 3 meses após a inserção. Os pacientes foram avaliados por até 5 anos após o término da prótese. Os desfechos incluíram a avaliação da taxa de sobrevivência do implante, comprimento da coroa, reabsorção óssea, acúmulo de placa, sangramento na sondagem (BOP), profundidade de sondagem periodontal (PPD) e avaliação do perfil de impacto na saúde bucal (OHIP). Ao final do período de acompanhamento de 5 anos, três implantes $(6,3 \%)$ do grupo de carga imediata falharam durante o período de observação. A perda óssea foi significativamente menor no grupo de carregamento retardado em 
comparação com os implantes carregados imediatamente. No final do segundo ano, os valores de BOP eram maiores no grupo carregado imediatamente. Ao longo do período de observação, os valores de IP no grupo com carga imediata foram maiores. PPD aumentou de forma consistente e durante os primeiros 3 anos no grupo de carga imediata. Os implantes hort inseridos para substituição de um único dente na maxila posterior apresentaram resultados clínicos satisfatórios nos conceitos de carga imediata e tardia. No entanto, os implantes carregados imediatamente apresentaram uma perda óssea aumentada e valores de BOP mais altos. Conforme avaliado pela pontuação do OHIP, uma melhora subjetiva foi observada em ambos os grupos, sem diferenças significativas.

Gallina (2018) avaliou a variação da espessura da tábua óssea vestibular e da posição da margem gengival em implantes unitários instalados em alvéolos de extração e em rebordos cicatrizados ambos com carga imediata em região estética. Para isso distribuiu os indivíduos em dois grupos, sendo o grupo 1(n=7) tratados com extração do dente com técnica minimamente invasiva, instalação de implante cone morse (Neodent $\left.{ }^{\circledR}\right)$, preenchimento do "gap" com substituo ósseo xenógeno (Bio Oss®) e cimentação de coroa provisória. Os indivíduos do grupo 2 (n=3), em rebordos unitários edêntulos receberam implante cone morse (Neodent $\left.{ }^{\circledR}\right)$ e cimentação de coroa provisória. Através de tomografia computadorizada, as medidas da espessura da tábua óssea vestibular (TOV) foram realizadas nos pontos coronal (C), médio (M) e apical (A) e, com auxílio de um guia de sondagem, as medidas da posição da margem gengival (PMG) foram realizadas nos pontos mesial (M), vestibular (V) e distal (D), ambas as análises foram aferidas no pré-operatório (T0), pós-operatório imediato (T1) e seis meses após a cirurgia (T2). Observou-se uma diferença estatística entre o tempo T0 e T2, sendo que o tempo T2 apresentou uma média das medidas maiores que no tempo T0 em ambos os grupos. O tratamento conferido no grupo 1 se mostrou opção confiável, com resultados de espessura do osso vestibular e nível gengival estáveis nos períodos observados, quando comparados com o tratamento do grupo 2. Com resultados similares entre os grupos, as vantagens do implante imediato como redução do tempo de execução do tratamento, redução da morbidade do pós-operatório, redução do uso de medicamentos e manutenção do arcabouço perimplantar se tornam atrativos para indicação da técnica testada.

Santana et al. (2018) relataram caso de paciente de 52 anos, do gênero feminino que apresentava falha de tratamento endodôntico/protético em região de incisivos central e lateral superiores. Ao exame físico inicial foi observada ponte fixa insatisfatória sobre as unidades 11 e 22. Ao exame de imagem foi constatada realização de tratamento endodôntico nas unidades 11 e 22, com lesão radicular adjacente, discreto aumento do espaço periodontal apical em ambas as unidades e fratura radicular da unidade 11. A paciente foi submetida à instalação imediata de implantes em área estética de maxila com customização de parafuso cicatrizador. A colocação de cicatrizador personalizado logo após a instalação dos implantes deve ser inerte ao tecido perimplantar e objetiva, principalmente, manter a arquitetura gengival até a realização da prótese definitiva, quando não é possível a carga imediata, como ocorreu neste caso. A personalização do parafuso cicatrizador deve ainda obedecer a uma excelente lisura superficial, a fim de evitar a retenção de restos alimentares e biofilme bacteriano, fatores que prejudicariam a cicatrização periodontal. A utilização do parafuso cicatrizador personalizado constitui-se como boa alternativa para manutenção da arquitetura gengival quando não é possível proceder à carga imediata.

Pol et al. (2018) em um estudo descritivo, longitudinal e prospectivo descreveram a evolução de 86 pacientes tratados com 99 implantes de unidade mediata com carga imediata precoce, colocados sobre osso cicatrizado. Na casuística, predominaram as mulheres entre 40-49 e 30-39 anos. A região anatômica mais implantada foi a maxila na região anterior, enquanto os critérios mais desfavoráveis foram: higiene bucal inadequada, tamanho do implante selecionado e não cumprimento das indicações estomatológicas. A evolução dos integrantes da série foi avaliada como satisfatória em 86,1\% do total. Um diagnóstico preciso, uma técnica cirúrgica cuidadosa e um tratamento protético correto favorecem o bom prognóstico deste procedimento. Salienta-se que a evolução e manutenção dos resultados dependerão da higiene bucal dos pacientes, bem como do estrito cumprimento das indicações estomatológicas e do comparecimento regular aos controles clínicos 
estabelecidos.

Mota (2018) revisou a literatura especializada e ilustrou com imagens um paciente submetido à técnica do implante imediato seguida de uma coroa provisória imediata. A técnica do implante imediato juntamente com a coroa provisória imediata tornou-se uma opção bastante segura para a reabilitação oral. Possui as vantagens de manter a arquitetura gengival perimplantar e reduzir o tempo e custo de trabalho, pois reduz os procedimentos cirúrgicos e a perda em altura e largura do osso alveolar. A coroa provisória imediata favorece a manutenção dos tecidos duro e mole da região, formando uma barreira entre o tecido duro que recebeu o implante beneficiando o sucesso dos casos. O biótipo gengival tem grande influência na estética no tratamento com implantes, sendo o mais fino com maior susceptibilidade a recessão gengival. Restaurações provisórias são importantes durante reabilitações orais com implantes, capazes de devolver a função de mastigação, fonética e estética do paciente, contribuindo também para a saúde e manutenção dos tecidos periodontais. A técnica do implante imediato à extração dentária é bastante viável e vantajosa, desde que bem planejada e cuidadosamente executada e o uso de coroa provisória imediatamente após a instalação de implante imediato proporciona bem-estar psicológico, representando uma solução reabilitadora para a perda de dente unitário anterior.

Batista (2019) avaliou o comportamento dos tecidos perimplantares (moles e duros) após a carga imediata de implantes unitários em áreas estéticas de maxila através de uma revisão de literatura. O comportamento dos tecidos perimplantares em implantes com carga imediata foi muito semelhante aos observados em implantes com carga tardia. Em maiores períodos de acompanhamento, foi verificado que as papilas podem migrar coronalmente ao longo do tempo e que os tecidos duros apresentaram perda óssea média de 1,0 mm, independentemente da utilização ou não de carga imediata. No que diz respeito à estética, em geral, os pacientes ficaram satisfeitos e as perdas notadas ao longo do tempo parecem não interferir da satisfação estética do paciente. Assim sendo a perda de papilas, recessão gengival vestibular e perda óssea podem ocorrer silenciosamente, sendo o tempo mais importante para sua ocorrência que a utilização de carga imediata ou não, o que torna a carga imediata uma alternativa viável e promissora em regiões estéticas sem comprometer o comportamento dos tecidos perimplantares.

Cruz et al. (2019) discutiram o caso clínico de uma paciente de 44 anos que apresentou perda do dente 21, após avaliação periodontal, radiográfica e tomográfica do elemento, foi realizado implante dentário através de um guia cirúrgico e prótese de acrílico curável por calor, em seguida instalada a carga imediata onde o provisório foi moldado com contorno subgengival côncavo. Nas marcações de avaliação, os tecidos perimplantares apresentaram-se estáveis e a paciente não relatou sintomas. Depois de selecionar o caso corretamente, o carregamento imediato do implante pode ser escolhido como um alternativa segura ao protocolo de convencional para a reabilitação de um único dente perdido na maxila, este procedimento otimiza tempos aguardando a reabilitação final, pois exigem menos intervenções cirúrgicas. Com a preparação adequada do perfil de emergência do provisório imediato, seguindo critérios específicos que marcam os contornos críticos e subcríticos é obtida cicatrização adequada dos tecidos perimplantares, resultando em um manuseio correto de tecidos duro e mole, que oferece um benefício biológico, funcional e estético para o paciente.

Soares (2019) analisou as vantagens e as taxas de sucesso na técnica dos implantes com carga imediata e provisionalização em única sessão. A instalação de implantes pós exodontia com carga imediata em única sessão reduz o tempo total de tratamento, os custos e a morbidade. Porém questões importantes ainda precisam de consenso na literatura tais como: manutenção do perfil de tecido ósseo e mucoso, estabilidade primária mínima necessária, e o entendimento dos motivos que levaram aos insucessos.

Sousa e Raucsh (2019) discutiram um caso de implante tardio, onde a técnica de implante imediato comprometia o prognóstico do caso, devido a possível infecção associada a fratura radicular do incisivo central superior direito (elemento 11), que era reabilitado com prótese fixa em metalocerâmica sobre pino metálico fundido. Devido a infecção instalada nesse 
alvéolo, o procedimento realizado foi a exodontia do elemento 11 fraturado, e a reabilitação mediata (tardia), com enxertia e provisionalização imediata. O procedimento cirúrgico de implante unitário tardio com carga imediata em região estética, é um procedimento muito bem indicado e com boa aceitação pelos pacientes. No caso de infecção associada a fratura radicular, a técnica mais bem aceita é a do implante tardio, após cicatrização dos tecidos, pois a enxertia com biomaterial e instalação do implante imediato em alvéolo possivelmente contaminado, podem prejudicar o sucesso do procedimento.

Costa et al. (2019) relataram caso de paciente do sexo feminino, 53 anos com fratura obliqua do dente 21, com necessidade de resolução imediata devido ao afastamento do domicilio e baixa situação econômica. O paciente foi totalmente avaliado pela equipe de trabalho e passou por avaliação clínico-radiográfica para atender aos requisitos principais estabelecido como a ausência de processo infeccioso e estado de saúde sistêmico, sendo indicado a colocação da implantação imediata à extração dentária sem confecção de retalhos, utilizando parafuso de titânio. Para a aplicação desta técnica, é necessária uma avaliação clínico-radiográfica correta e participação ativa do paciente no cuidado indicado pós-operatório, pois, embora seja um técnica eficaz, requer mais cuidados pós-operatórios para evitar suas falhas.

Lucas et al. (2019) compararam o índice de sucesso e previsibilidade à curto prazo de implantes imediatos instalados em regiões anterior e posterior em 43 prontuários sendo anterior $(n=20)$ e posterior $(n=23)$. Foram incluídos aqueles com indicação de extração dentária, instalação de implantes imediatos unitários, no mínimo doze meses de segmento com implante funcional. Os critérios de sucesso foram baseados na escala de saúde dos implantes dentários do Congresso Internacional de Implantodontia Oral, eixo I. e II.: ausência de dor, ausência de mobilidade, ausência de exsudato e perda óssea de até $4 \mathrm{~mm}$. Valor de $\mathrm{p}<0.05$ foi considerado estatisticamente significante. O índice de sucesso dos implantes imediatos foi de 97,7\% para implantes em função por pelo menos 12 meses. $\mathrm{O}$ uso de biomaterial $(\mathrm{p}=0,03)$ e prótese provisória $(\mathrm{p}<0,0001)$ foi significantemente maior em região anterior. Não foi encontrado diferença significante quanto a falha dos implantes comparando os dois grupos $(\mathrm{p}=0,47)$. Não houve diferença estatisticamente significante entre os grupos, considerando a idade, gênero, motivo da extração, torque inicial, tempo de tratamento e tipo de plataforma do implante $(\mathrm{p}>0,05)$. As regiões anterior e posterior apresentaram alta taxa de sucesso a curto prazo quanto a técnica de implante imediato.

Sampaio et al. (2020) evidenciaram o sucesso de implante dental unitário, instalado imediatamente após exodontia e curetagem de lesão periapical extensa, e a instalação da prótese com proservação de 33 meses ao relatar o caso de paciente do sexo feminino, 24 anos de idade, com uma parúlide na gengiva inserida acima do dente 25 e fratura coronal do mesmo. TCFC revelou uma extensa área hipodensa na região do ápice da raiz do dente 25, medindo 6,7 X 8,2 mm. A referida lesão já havia causado fenestração da parede vestibular, cerca de dois terços no sentido ápice-coroa. Foi instituído como plano de tratamento a instalação de implante dentário imediato com carga imediata. A prótese final foi instalada 13 meses após a instalação do implante. Decorridos 17 meses do procedimento cirúrgico, foi solicitada radiografia periapical de controle, onde foi possível avaliar trabeculado ósseo sadio em torno do implante. O avanço dos biomateriais utilizados em reabilitação oral e o aprimoramento das técnicas e profissionais que a desempenham, torna possível realizar implantes imediatos e reabilitação imediata em área infectada, mesmo em casos limítrofes, com segurança e efetividade, possibilitando a obtenção de resultado final satisfatório.

Sierra-Rebolledo e Jimenez-Tortolero (2020) se propuseram a determinar as dimensões da crista óssea vestibular dos incisivos mandibulares com indicação de implantação imediata através de um estudo transversal realizado em pacientes com necessidade de colocação de implantes de unidades únicas imediatas na área dos incisivos superiores. Cortes tomográficos sagitais foram usados para determinar altura e espessura da crista óssea alveolar vestibular. O ponto de medição de espessura estava localizado $4 \mathrm{~mm}$ apical à linha amelocementária. Uma análise T-student foi usada para comparar variáveis de acordo com idade, sexo e grupo dentário, com um intervalo de confiança de 95\%. Foram avaliadas 298 imagens. A altura média foi de $10,68 \mathrm{~mm}$, não houve diferenças ao comparar o grupos. A espessura média foi de $0,73 \mathrm{~mm}$, diferenças de espessura 
estatisticamente significativas, foram observadas na comparação de idade e sexo, mas não no grupo dentário A altura do osso alveolar vestibular dos incisivos superiores é suficiente para colocar implantes imediatos dentro de uma estrutura óssea.

Bitar Júnior et al. (2020) realizaram uma revisão bibliográfica de literatura, sobre implantação dentária imediata em sítio contaminado com subsequente provisionalização de forma a trazer ao debate este tema pertinente para a atual odontologia. O procedimento é viável para a reabilitação funcional e estética de pacientes que possuem elementos dentários com indicação para a exodontia devido infecção bacteriana local.

Falcon-Guerrero e Falcon-Pasapera (2020) executaram uma revisão bibliográfica para determinar quais considerações devem ser necessárias para instalar um implante imediato nos alvéolos com processos infecciosos. É possível obter os benefícios dos implantes imediatos em alvéolos infectados após extração dentária, desde que a aplicação de um manejo clínico-cirúrgico adequado, quando o tratamento com antibióticos apropriado é considerado, acompanhado de desbridamento e desinfecção alveolar adequados, respeitando a cadeia de assepsia e antissepsia, posição subcrestal do implante para obter a estabilidade primária do implante. Agregados plaquetários ricos em fatores de crescimento são sugeridos para melhorar o prognóstico deste tratamento alternativo.

A reabilitação de pacientes desdentados é um processo complexo, em que devem ser considerados aspectos fisiológicos, patológicos e psicológicos, entre eles, o envelhecimento, a alteração do fluxo salivar, a redução da coordenação motora, a reabsorção óssea do rebordo, a fragilidade do tecido mucoso, as alterações dos tecidos de suporte e a adaptação psicológica. Devido à sua interferência, devem ser considerados quando se planeja uma reabilitação oral com implantes (Farro, 2017).

Implantes pós-extrativos imediatos são aqueles implantes colocados imediatamente após a extração do dente em um alvéolo de extração fresco A principal vantagem do procedimento é encurtar a duração do tratamento uma vez que não temos que esperar pela cura do tecido mole ( 2 a 6 semanas) ou para consolidação óssea (4 a 6 meses), no entanto, implantes pósextrativos imediatos podem ter maior risco de complicações e falhas (Felice et al., 2015).

Costa (2018) afirma que implantes osseointegrados são indicados em casos de edentulismos parciais e totais, confecção de elementos unitários, edentulismos com distribuição desfavorável ou número insuficiente de pilares naturais para próteses fixas, insatisfação ou rejeição às próteses totais ou parciais removíveis.

Entre as contraindicações relativas ao paciente estão as doenças sistêmicas não controladas, uso de medicação crônica, radioterapia localizada sobre a região edêntula, pacientes em crescimento, gravidez, expectativas não-realísticas sobre o tratamento, bruxismo, higiene oral inadequada, hábitos como fumar e beber. Pacientes que fazem uso de nicotina, álcool, ou drogas, podem ter um efeito negativo sobre vascularização do sítio cirúrgico exigindo cautela ao planejar implantes para esses indivíduos. Contraindicações também dizem respeito ao profissional, seu treinamento ou fata dele e sua experiência com implantes (Costa, 2018).

Para os autores Grandi et al. (2015), Felice et al. (2015), Guidetti et al. (2015) as contraindicações abrangeram pacientes imunossuprimidos ou imunocomprometidos, tratamento intravenoso com amino-bifosfonatos, os diabéticos não controlados, gestantes ou lactantes, pacientes com problemas psiquiátricos, má higiene oral e falta de motivação, periodontite não tratada, infecção ou inflamação aguda na área destinada à colocação do implante, necessidade de aumento ósseo na inserção do implante, falta de dentição ou prótese oclusiva oposta.

Assis e Araújo (2016) afirmaram que para um resultado satisfatório com implantes, é importante obedecer às corretas indicações quais sejam o travamento primário, boa condição óssea, escolha adequada do implante, condição sistêmica satisfatória do paciente, ausência de maus hábitos como tabagismo e bruxismo.

A reposição de elementos dentais na região anterior envolve, principalmente, o aspecto estético conforme afirma Matsumoto (2016). A integração óssea entre osso e implante é a chave do sucesso cirúrgico, devendo ser observados alguns 
fatores para que o procedimento ocorra de maneira satisfatória, entre eles a altura óssea e gengival, sendo necessária uma análise criteriosa do local pré-operatório, da técnica cirúrgica e dos métodos utilizados no planejamento do procedimento para uma correta localização do implante e restauração subsequente (Matiello e Trentin, 2015). Outros fatores a serem observados são a extração dentária a traumática, preservando o tecido ósseo e arquitetura gengival, além da estabilidade primária, evitando qualquer contato oclusal do implante conforme os autores (Tanaka Júnior, 2015; Andreiuolo et al. 2016; Grandi et al., 2015; Mota, 2018).

Como observaram Miguel Júnior et al. (2016) o preenchimento do espaço alveolar deve ser realizado com o propósito de ser evitada uma possível reabsorção óssea da parede alveolar, comprometendo o resultado final. A utilização de próteses livres de metal é uma opção viável, do ponto de vista estético e funcional, suprindo a necessidade do paciente. O diâmetro do implante deve ser o mais compatível possível com o diâmetro da raiz a ser extraída, com a finalidade de preencher o melhor possível o alvéolo, evitando a presença de grandes espaços entre o implante e a parede alveolar (Miguel Júnior et al., 2016).

Para determinar a taxa de sucesso dos sistemas de implantes e próteses, é importante avaliar parâmetros clínicos, radiológicos e de estabilidade conforme asseguram Guideti et al. (2015). Conforme os autores, entre os parâmetros clínicos que podem ser utilizados para avaliar o estado da mucosa perimplantar, a profundidade de sondagem é a mais precisa, mas nem sempre o aumento da profundidade de sondagem representa perda óssea. Os implantes são geralmente considerados bem sucedidos quando apresentam uma profundidade de sondagem menor ou igual a 3 milímetros. (Guideti et al., 2015).

Para Soares (2019) é importante avaliar se a quantidade de tecido ósseo na porção apical do alvéolo será suficiente para alcançar a estabilidade primária adequada. Sierra-Rebolledo e Jimenez-Tortolero (2020) postulam que dimensões do osso alveolar são decisivas no planejamento e prognóstico dos implantes imediatos. O comprimento do implante, regeneração óssea e durabilidade dos resultados clínicos, dependem de uma crista ideal em termos de altura e espessura.

Guideti et al. (2015), Matsumoto et al. (2016) consideram valores de estabilidade primária do implante são considerados aceitáveis entre 56,5 N.cm e $45 \mathrm{Ncm}$ para o carregamento funcional imediato. No entanto, Soares (2019) observa que não há consenso na literatura quanto ao torque mínimo de inserção exigido. Para Matsumoto et al. (2016) as características quanto à forma e superfície do implante favorecem a estabilidade primária, a redução da micromovimentação e melhora da posição do osso sobre a superfície do implante e favorece a cicatrização no local, contribuindo para o sucesso do tratamento.

Sierra-Rebolledo e Jimenez-Tortolero (2020) consideram que para os incisivos superiores a altura da crista óssea vestibular parece ser suficiente para a colocação dos implantes dentro de uma estrutura óssea quando uma espessura menor que $1 \mathrm{~mm}$ deve ser esperada na maioria dos casos, indicando a necessidade de aplicação de técnicas de regeneração tecidual guiada, principalmente em situações de implante imediato e onde a estética é um fator determinante.

Estudos comparativos realizados por Den Hartog et al. (2016), Tonetti et al. (2017), Heydecke et al. (2018), Ayna et al. (2018), Grandi et al. (2015), Felice et al., (2015) entre carregamento imediato e carregamento convencional de implantes de dentes unitários não mostraram diferenças significativas em termos de perda óssea marginal, complicações, aspectos de partes moles, resultado estético e satisfação do paciente.

Lucas et al. (2019) encontrou semelhança quanto aos altos índices de sucesso e previsibilidade à curto prazo de implantes imediatos instalados nas regiões anterior e posterior. Mota (2018) afirma que na região anterior da maxila, onde a estética é um fator importante, os implantes imediatos promovem a diminuição do período de cicatrização e os tecidos duros e moles que os circundam, sendo importante observar o biótipo gengival. O mais fino é mais susceptível à recessão gengival, deiscência e fenestrações (Mota, 2018).

Tonetti et al. (2017) verificaram que o fechamento primário ideal foi obtido com mais frequência em implantes tardios, os quais também eram menos propensos a exibir falha da ferida. As profundidades de sondagem em torno dos implantes imediatos foram maiores do que em implantes retardados no momento da inserção da coroa e aos 12 meses assim 
como níveis mais elevados de perda óssea radiográfica durante o acompanhamento de 36 meses, o que sugere que as diferenças podem ser confundidas por uma colocação mais profunda de implantes imediatos na hora da cirurgia. Os implantes imediatos resultaram em uma recessão marginalmente menor do tecido mole em torno dos dentes adjacentes $0,3 \mathrm{~mm}$ vs 0,5 mm, mas esse achado não atingiu significância estatística. Os implantes retardados parecem ter melhor resultados estéticos do que implantes imediatos, de acordo com a pontuação PES.

Em outro estudo realizado por Ayna et al. (2018), pacientes foram avaliados por até 5 anos após o término da prótese e observou-se a perda óssea e os valores de sondagem periodontal maiores nos implantes carregados imediatamente do que nos implantes convencionais. Já na avaliação do perfil de impacto na saúde bucal, não ocorreram diferenças significativas (Ayna et al., 2018).

Quanto à sobrevivência dos implantes, o resultado da carga imediata não é inferior à carga convencional para implantes de dentes unitários anteriores colocados em locais cicatrizados segundo Den Hartog et al (2016). Taxas de 83,55\% após 12 de meses da implantação foram obtidas por Guideti et al. (2015).

Guideti et al. (2015), Pol et al. (2018) afirmaram que a carga funcional imediata de implantes unitários na área posterior da mandíbula é considerada uma técnica confiável. Para isso, é importante que o paciente não apresente bruxismo, possua osso suficiente para o apoio e que uma boa higiene oral seja praticada para obter uma boa taxa de sobrevida do implante.

Mota (2018) discorreram quanto às principais limitações de se confeccionar uma prótese imediata. Uma delas é a técnica muito precisa, que exige que o cirurgião-dentista assegure que não haja movimentação do implante durante a osseointegração, indicação da técnica apenas em situações específicas, o implante deve suportar torque de $35 \mathrm{Ncm}$, infecções locais ou estresse excessivo não previsto.

Sobre as tomografias computadorizadas de feixe cônico Araújo et al. (2018) afirmaram que são ferramentas viáveis para a região maxilofacial, pois permitem a mensuração de diversos parâmetros de tecido mole e duro da cavidade bucal, úteis para o planejamento e previsibilidade de tratamento reabilitador com implantes dentários. No entanto, diante de limitações das imagens radiográficas convencionais, as imagens tridimensionais vêm ganhando espaço por permitir imagens sem ampliação e dimensões reais, o que favorece um correto diagnóstico. Possuem vantagens como os modos de exibição exclusivos para imaginologia maxilofacial, limitação do feixe de Raios X, exatidão de imagem, nitidez da imagem, scanner rápido, dose reduzida de radiação (Araújo et al., 2018).

Santana et al. (2018) afirmaram que a colocação de cicatrizador personalizado logo após a instalação do implante objetiva, principalmente, manter a arquitetura gengival até a realização da prótese definitiva, quando não é possível a carga imediata. Os autores ressaltam que cicatrizador deve ter uma excelente lisura superficial, para evitar a retenção de restos alimentares e biofilme bacteriano, o que prejudicaria a cicatrização periodontal (Santana et al., 2018).

Sousa e Rausch (2019) apontaram que no caso de infecção associada a fratura radicular, a técnica mais bem aceita é a do implante tardio, após cicatrização dos tecidos, pois a enxertia com biomaterial e instalação do implante imediato em alvéolo possivelmente contaminado, podem prejudicar o sucesso do procedimento.

Mesmo em sítios infectados, conforme os autores Bitar Júnior et al. (2020), Falcon-Guerrero e Falcon-Pasapera (2020), Sampaio (2020) a sobrevida dos implantes imediatos pode ser bastante satisfatória e apresentar taxas de sobrevivência próximas dos implantes colocados em locais saudáveis. O melhor prognóstico dependerá de vários procedimentos do tratamento incluindo a assepsia e antissepsia, prescrição adequada de antibióticos sistêmicos profiláticos, desbridamento minucioso do alvéolo após extração dental minimamente invasiva, e gerenciamento eficaz da carga imediata até a restauração definitiva (Bitar Júnior et al., 2020; Falcon-Guerrero \& Falcon-Pasapera, 2020; Sampaio, 2020).

O uso de implantes para melhorar a função, estética e saúde bucal avançou a partir do conceito de osseointegração, 
evoluiu de protocolos convencionais com duas fases cirúrgicas para implantação imediata após a exodontia, inclusive com carga imediata, possibilitando a reabilitação oral de edêntulo totais, parciais e de unitários. Contribuindo para a manutenção da papila, favorecendo a estética rosa.

\section{Considerações Finais}

A literatura é ampla e demonstra altas taxas de sucesso e previsibilidade de implantes com carga imediata, sendo um procedimento muito benéfico para o paciente devido à redução de tempo total de tratamento, redução de custos e resultados bastante satisfatórios, inclusive quando se trata de estética e satisfação do paciente. No entanto, o uso de implantes com carga imediata requer planejamento cuidadoso.

\section{Referências}

Andreiuolo, R., Vasconcellos, F., Andrade, A., Groisman, M. \& Vidigal Júnior, G.M. (2016). Implante imediato na região anterior: aspectos cirúrgicos e protéticos. Revista Brasileira de Odontologia, 73 (1), 84-8.

Araújo, L. N. M. de, Mendes, D. L. V., Santos, M. T. dos, Carvalho, R. D. de, Dantas, E. M., Calderon, P. dos S. \& Gurgel, B. C. de V. (2018). Caracterização tomográfica das áreas edêntulas previamente à reabilitação com implantes unitários. Rev Odonto, 47 (4), $219-216$.

Assis, L. C. de \& Araújo, M. O. (2016). Carga imediata em implantodontia: revisão de literatura. Trabalho de Conclusão de Curso (TCC) em Odontologia. UNIT.

Ayna, M., Wessing, B., Gutwald, R., Neff, A., Ziebart, T., Açil, Y., Wiltfang, J. \& Gülses, A. (2018). A 5-year prospective clinical trial on short implants (6 $\mathrm{mm}$ ) for single tooth replacement in the posterior maxilla: immediate versus delayed loading. Odontology. https://doi.org/10.1007/s10266-018-0378-x

Batista, S. H. B. (2019). Comportamento dos tecidos moles em redor de implantes com carga imediata: revisão literária. Mestrado em Medicina Dentária, Faculdade Ciências da Saúde. https://bdigital.ufp.pt/bitstream/10284/9047/1/PPG_38846.pdf

Bitar Júnior, B. J. G., Silva, S. A. C. da, Araújo, T. B. G., \& Corrêa, A. K. M. \& Camilotto, L. S. (2020). Implante e Provisionalização Imediatos em Alvéolo Infectado: revisão de literatura. Braz. J. of Develop, 6 (12), 94695-94705

Costa. T. C. (2018). Pré-requisitos iniciais em um planejamento de reabilitação oral com implantes. Faculdade de Odontologia Universidade Federal de Minas Gerais. Doi http://hdl.handle.net/1843/ODON-B3GJH9

Cruz, A. M. A., Soto, Y. M., Gutierrez, A. E. P., \& Sanchez-Marin, C. G. (2019). Manejo de tejidos blandos en implante con carga imediata del sector anterosuperior: reporte de caso clínico. Revista ADM, 79 (3), 169-172.

Den Hartog, L., Raghoebar, G. M., Stellingsman, k., Vissink, A. \& Meijer, H. J. A. (2016). Imemdiate Loading of anterior single-tooth implants placed in healed sites: five-year results of a randomized clinical trial. The Internetional Journal of Prosthodontics, 29 (6). https://doi.org/10.1007/s10266-018-0378

Falcón-Guerrero, B. E. \& Falcón-Pasapera, G. S. Consideraciones para el manejo de un implante inmediato en alvéolos infectados: una actualización. Revista ADM, 77 (3), 155-161.

Farro, C. (2017). Implantes em carga imediata pós-extração: revisão bibliográfica. Mestrado em Medicina Dentária. Instituto Universitário de Ciências da Saúde.

Felice, P., Trullenque-Eriksson, A., Barausse, C. \& Esposito, M. (2015). Immediate non-occlusal loading of immediate post-extractive versus delayed placement of single implants in preserved sockets of the anterior maxilla: 1- year post-loading outcome of...Eur J Oral Implantol, 8(4), 361-372.

Galina, K. (2018). Implantes imediatos com carga imediata em região estética: ensaio clínico controlado. Mestrado em Odontologia. Centro de Ciências Biológicas e da Saúde, Universidade Estadual do Oeste do Paraná.

Grandi, T., Hant, T., Samarani, R. \& Samarani, L. (2015). Immediate, early (3 weeks) and conventional loading (4 months) of single implants: Preliminary data at 1 year after loading from a pragmatic multicenter randomised controlled trial, Eur J Oral Implantol, 8 (2), $115-126$.

Guidetti, L. G. C., Monnazzi, M.S., Piveta, A. C. G., Gabrielli, M. A. C., Gabrielli, M. F. R., Pereira Filho V. A. \& Gercke M. G. (2015). Implantes unitários instalados na região posterior mandibular sob carga imediata: estudo prospectivo. Unesp. http://hdl.handle.net/11449/131190

Heydecke, H., Mirzakhanian, C., Behneke, A., Behneke N., Fügl, A., Zechner. W., Baer, R.A., Nölken, R., Gottesman, E., Colic, S., Ottria, L. \& Pozzi, A. (2015). A prospective multicenter evaluation of immediately functionalized implants: Preliminary data at 1 year after loading from a pragmatic multicenter randomised controlled trial. Eur J Oral Implantol, 8 (2), 115-126.

Lucas, R.R.S., Martins, C. C. P., Oliveira, H. E., Cordeiro, B. Q. da S., Machado, A. N., Casado, P. L., Aguiar, T. R. da S. \& Tristão, G. C. (2019). Evaluation of the success rates of immediate implant placed in anterior and posterior regions: a retrospective study. Dental Journal, 4 (3).

Marconi, M. A. \& Lakatos, E. M. (2007). Técnicas de pesquisa: planejamento e execução de pesquisas, amostragens e técnicas de pesquisas, elaboração, análise e interpretação de dados. (6a ed.), Atlas. 
Research, Society and Development, v. 10, n. 11, e237101119546, 2021

(CC BY 4.0) | ISSN 2525-3409 | DOI: http://dx.doi.org/10.33448/rsd-v10i11.19546

Matiello, C. N. \& Trentin, M. S. (2015) Implante dentário com carga imediata na região anterior superior: relato de caso clínico. RFO, 20 (2), 238-242

Matsumoto, W., Hotta, T. H., Antunes, R. P. de A. \& Reino, D. M. (2016). Implante unitário anterior procedimentos de enxertia e provisionalização: relato de caso. Revista Bahiana de Odontologia, 1(63), 63-73. 2238-2720revbahianaodonto.v7i1.765

Miguel Júnior, H.; Genevose, W. J., Beltrão, C. F. B., Kassardjian, F., \& Cerri, A. (2016). Implante imediato associado ao enxerto de tecido conjuntivo: relato de caso clínico. Revista Associação Paulista de Cirurgião Dentista, 70 (3), 312-6.

Mota, A. R. (2018). Provisionalização imediata após instalação de implante imediato em área estética: revisão de literatura. Especialização em Prótese Dentária. Faculdade de Odontologia, Universidade Federal de Minas Gerais.

Pol, M. M.; Samalea, L. P., Samalea, L. P., Garcia, S. C. \& Estrada, M. L. D. de. (2018). Evolución de pacientes rehabilitados con implantes mediatos unitários de carga inmediata temprana de tipo Leader Implus. MEDISAN, 22 (2), 112.

Sampaio, V. P. R., Silva, D., F., B., Barreiro, F. M. P., Brito, H. B. S., Andrade, F. J. P. de. \& Gomes, D. Q. de C. (2020). Implante imediato associado a enxerto xenógeno e provisionalização imediata em área infectada: relato de caso. Arch Health Invest, 9 (5), 444-448. http://dx.doi.org/10.21270/archi.v9i5.4784

Santana, D. C. P., Dultra, F. K. A. A., Dultra, J. de A., Oliveira, F. \& Correa, M. P. (2018). Cicatrizador personalizado em implantes imediatos: relato de caso. Revista Odontológica de Araçatuba, 39 (2), 09-12.

Sierra-Rebolledo, A. \& Jimenez-Tortolero, R. (2020). Dimensiones de la cresta ósea vestibular en incisivos maxilares con indicación de implantes inmediatos. Un estudio transversal y sus implicaciones en el plan de tratamiento. Int. J. Inter. Dent, 13 (2), 71-75.

Silva, da M. C. \& Almeida, de S. B. (2017). Implante imediato com reabilitação imediata. Trabalho de Conclusão de Curso (TCC) em Odontologia, Centro Universitário São Lucas.

Soares, L. J. (2019). Técnica de exodontia, instalação de implante e provisionalização imediata em sessão única. Trabalho de Conclusão de Curso (TCC) em Odontologia. Universidade Federal de Sergipe.

Souza, L. dos S. de. \& Rausch, F. Z. (2019). Implante unitário com provisionalização imediata: relato de caso clínico. Revista UNINGÁ, 56 (3), $101-112$.

Tanaka Júnior, H. (2015). Implante unitário com carga imediata. Trabalho de Conclusão de Curso (TCC) em Odontologia. Departamento de Odontologia da Faculdade de Ciências da Saúde da Universidade de Brasília.

Tonetti, M. S., Cortellni, P., Graziani, F., Cairo, F., Lang, N. P., Abundo, R. Conforti, G. P., Marquardt, S., Rasperini, G., Silvestri, M., Wallkamm, B. \& Wetzel, A. (2017). Immediate versus delayed implant placement after anterior single-tooth extraction: the timing randomised controlled clinical trial. Journal Clinical Periodontology, 44, 215-224

Trentini, M. \& Paim, L. (1999). Pesquisa em Enfermagem. Uma modalidade convergente-assistencial. Editora da UFSC. 